\title{
Diagnosi microbiologica dell'infezione dell'emergenza cutanea e del tunnel nei pazienti in CAPD
}

\author{
D. Crotti ${ }^{1}$, M.C. Medori ${ }^{1}$, M.R. Caruso ${ }^{2}$, U. Buoncristiani ${ }^{2}$ \\ ${ }^{1}$ Servizio di Microbiologia Clinica e $e^{2}$ U.O. di Nefrologia e Dialisi, \\ Azienda Ospedaliera di Perugia
}

ei pazienti in dialisi peritonea-

le ambulatoriale continua (CAPD), il problema cardine nella gestione del paziente è l'adeguata sorveglianza delle infezioni al medesimo collegate (1-5). Accanto agli aspetti preventivi (6-9) sovente aleatori (10), una corretta diagnosi di infezione è altresì momento essenziale onde garantire $\mathrm{i}$ più adeguati interventi terapeutici (11-13). Le conseguenze più temute a tutto ciò sono, infatti, la peritonite, la necessità di una rimozione del catetere, con tutti i suoi risvolti logistici, e, nell'impossibilità di una sua sostituzione, il ricorso all'emodialisi $(14,16)$.

In particolar modo è l'infezione dell'emergenza cutanea ("exit-site infection", ESI) che presenta i più rilevanti problemi di attenzioni anche da un punto di vista strettamente microbiologico (1, 3-5, 17). Per emergenza cutanea ("exit-site" in lingua inglese) si intende il punto in cui il catetere emerge dalla cute, ovvero la parte di cute che circonda e delimita il foro cutaneo da cui emerge il catetere peritoneale $(13,15,17)$. Il tramite ("tunnel" in lingua anglosassone, termine che noi stessi abbiamo adottato in tale presentazione) è invece il percorso sottocutaneo del catetere dalla cuffia preperitoneale (compresa) fino a circa $2 \mathrm{~cm}$ dal foro di uscita cutaneo del catetere medesimo (13), ovverosia il tragitto sottocutaneo del catetere peritoneale dalla cute alla cavità peritoneale (Fig. 1).
In condizioni fisiologiche l'emergenza è asciutta, pulita, non arrossata, senza croste, non dolente né pruriginosa. Il catetere, per contro, è palpabile, con maggiore o minore facilità a seconda della quantità di grasso sottocutaneo, non è dolente, la cute sovrastante è di colore e temperatura normali $(13,15)$.

Il sospetto di infezione nasce nel momento in cui compaiono, a volte sfumati e incompleti, i segni tradizionali della flogosi (17). Dalla diagnosi clinica l'iter operativo per una diagnosi microbiologica e, quindi, per una corretta diagnosi eziologica di infezione, è quanto mai importante e delicato $(5,11,13)$.

In tale presentazione riportiamo dapprima i risultati relativi alla nostra esperienza, riferita retrospettivamente agli anni 1995-96; quindi, sulla base di iniziali valutazioni prospettiche condotte nel 1997, delineiamo una proposta di linee guida cui potersi attenere, per una corretta, tempestiva e razionale diagnosi eziologica di infezione, sia dell'emergenza cutanea come del tunnel del catetere intraperitoneale.

\section{Esperienza personale}

Nel corso del biennio 1995-96 abbiamo potuto elaborare $\mathrm{i}$ dati relativi a 25 casi di infezione dell'emergenza cutanea (non altrettanto dicasi per le infezioni del tunnel, di cui non siamo in grado di fornire rag- guagli dettagliati). Come riportato in Tabella I, hanno prevalso i Gram-positivi (56\%), con Staphilococcus aureus decisamente predominante ( $48 \%$ dei casi) $(2$, $3,5,7)$. Solo in parte può sorprendere la frequenza, seconda in ordine di incidenza, di infezioni apparentemente sostenute da Pseudomonas aeruginosa (20\%) (1, 2, 4, 12).

Dato l'esiguo numero di casi relativo ai singoli ceppi microbici isolati, possiamo

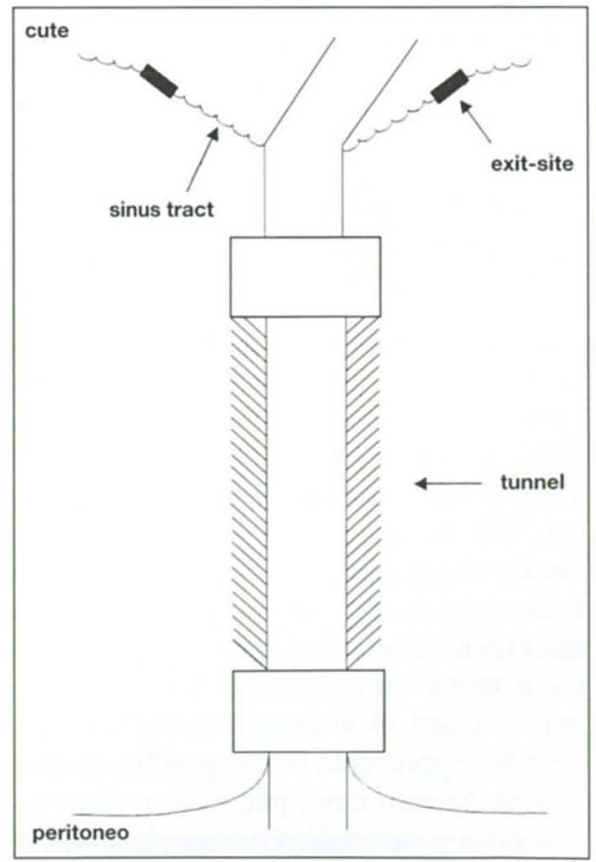

Fig. 1 - Anatomia dell'emergenza cutanea (exit-site) e del tramite (tunnel) 
TAB. I - RISULTATI COMPLESSIVI RELATIVI ALLE INFEZIONI DELL'EMERGENZA CUTANEA NEL BIENNIO 1995-96

\begin{tabular}{|c|c|c|c|c|}
\hline \multirow{2}{*}{$\begin{array}{l}\text { Eziologia } \\
\text { Gram-positivi }\end{array}$} & \multirow{4}{*}{$\begin{array}{l}\text { Staphylococcus aureus } \\
\text { staphylococcus epidermidis } \\
\text { Staphylococcus sp }\end{array}$} & \multicolumn{3}{|c|}{ Emergenza cutanea } \\
\hline & & 12 casi $\bullet^{\circ}$ & $48 \%$ & \\
\hline & & 2 casi $x$ & $8 \%$ & $(4 \%)$ \\
\hline & & 1 caso & $4 \%$ & \\
\hline & Streptococcus sp & 1 caso $\bullet$ & $4 \%$ & $(0 \%)$ \\
\hline & corineforme & 1 caso $x$ & $0 \%$ & $(4 \%)$ \\
\hline \multirow[t]{2}{*}{ Gram-negativi } & Pseudomonas aeruginosa & 5 casi & $20 \%$ & \\
\hline & Escherichia coli & 2 casi ${ }^{\circ}$ & $8 \%$ & $(4 \%)$ \\
\hline \multirow[t]{2}{*}{ Funghi } & Candida albicans & 1 caso & $4 \%$ & \\
\hline & Candida tropicalis & & $4 \%$ & \\
\hline
\end{tabular}

TAB. II - "PATTERNS" DELLE RESISTENZE OSSERVATE IN S. AUREUS NEL PERIODO 1995-96 NEI CEPPI ISOLATI DA TAMPONE DELL'EMERGENZA

\section{2 ceppi di Staphylococcus aureus}

\begin{tabular}{|c|c|c|}
\hline Antibiotico & Ceppi resistenti & Percentuale \\
\hline $\begin{array}{l}\text { - meticillina } \\
\text { (B lattamici) }\end{array}$ & 4 & $34 \%$ \\
\hline $\begin{array}{l}\text { - gentamicina } \\
\text { (aminoglicosidi) }\end{array}$ & 5 & $42 \%$ \\
\hline $\begin{array}{l}\text { - ciprofloxacina } \\
\text { (chinoloni) }\end{array}$ & 4 & $34 \%$ \\
\hline - tetraciclina & 3 & $25 \%$ \\
\hline - rifampicina & 1 & $8 \%$ \\
\hline - eritromicina & 5 & $42 \%$ \\
\hline - cotrimoxazolo & 0 & - \\
\hline $\begin{array}{l}\text { - vancomicina } \\
\text { (teicoplanina) }\end{array}$ & 0 & - \\
\hline
\end{tabular}

riportare il "pattern" delle resistenze antimicrobiche esclusivamente per il predominante $S$. aureus. Ebbene, le resistenze (Tab. II) nei confronti di meticillina non sono smisuratamente elevate, attestandosi sul 34\%. Il dato è significativo (e importante), in quanto la resistenza a meticillina è espressione di resistenza ai Beta-lattamici (penicilline e cefalosporine), così come è strettamente correlata alla resistenza nei confronti di aminoglicosidi (gentamicina ne rappresenta la categoria) e di chinoloni. Nessun caso, poi, si è rivelato resistente a vancomicina e teicoplanina, così come a cotrimoxazolo (sicuramente più maneggevole dei glico-peptidi).
Dalla valutazione critica dei risultati osservati, abbiamo potuto rilevare come in 1 caso su 12 per $S$. aureus, in 1 su 2 per Staphilococcus epidermidis, nell'unico caso di Staphilococcus sp. (diverso dai precedenti) e nell'unico caso di Corinuti in laboratorio dal tampone prelevato dall'emergenza cutanea infetta o sospetta tale, gli schizomiceti in questione fossero stati isolati esclusivamente dopo arricchimento in brodo del medesimo e non dalla coltura diretta, come in tutti gli altri casi. Questo potrebbe significare un loro ruolo di contaminanti il prelievo medesimo, piuttosto che di reali responsabili di infeneforme, identificati sugli isolamenti otte- zione (quanto meno supposta).

$\mathrm{Nel}$ corso del 1997 abbiamo processato 11 campioni relativi ad altrettanti casi di presunta infezione (sospettata su base clinica). L'indagine era mirata essenzialmente a fornire criteri diagnostici il più coerenti possibile e a monitorare, conseguentemente, le resistenze antimicrobiche. In Tabella III sono riportati i risultati. Orbene, in 3 casi, soltanto la coltura dopo arricchimento in brodo triptosio ("over-night") diede esito positivo: 2 casi di $S$. epidermidis ed 1 di Enterococcus faecalis. In tutti i 3 casi si ipotizzò, a ragione, anche sulla base di un colloquio clinico, una contaminazione al momento del prelievo. In un quarto caso, dopo prearricchimento, crebbero 2 ceppi di stafilococco, di cui uno venne interpretato, per le stesse motivazioni (in quanto non cresciuto da coltura diretta), come contaminante (S. cohnii).

Va menzionato il fatto che in tutti i casi riportati non si osservarono resistenze degne di rilievo (vedi sempre Tab. III); in particolare, i ceppi di stafilococco sono risultati sempre sensibili a meticillina, quelli di enterococco ad ampicillina, quelli di Corynebacterium a vancomicina. Colpisce, in tale, peraltro limitata, casistica, l'assenza di Gram-negativi.

La disamina dei risultati riportati in tale lavoro, così come l'attenta valutazione di analoghe e/o sovrapponibili esperienze (anche personali) in simili o equivalenti campi di interesse clinico-microbiologico, ci hanno portato a delle conclusioni operative che riportiamo di conseguenza (18).

\section{Protocolli diagnostici}

L'infezione dell'emergenza cutanea come del tunnel peritoneale, viene sospettata clinicamente.

La diagnosi microbiologica consegue pertanto alla rilevazione clinica di un focolaio infettivo. Adeguati procedimenti preanalitici dapprima, così come standardizzate e congrue fasi analitiche successivamente, potranno garantire una verosimile diagnosi eziologica di infezione, tale da correlare clinicamente una meta-analisi dei risultati ottenuti in laboratorio.

\section{Emergenza cutanea}

La coltura dei materiali cutanei trova indicazione solo in presenza di segni di in- 
fezione locale (18). L'associazione di uno o più segni dell'infiammazione con la presenza di una secrezione più o meno torbida $o$ francamente purulenta è più che sufficiente per porre diagnosi clinica di infezione, e quindi intervenire con un prelievo e con adeguate metodologie analitiche nel laboratorio microbiologico.

\section{Fase pre-analitica}

Il tampone, eventualmente inumidito con soluzione fisiologica sterile, va stemperato sulla cute secernente che circonda l'emergenza del catetere. Il tampone va così inviato quanto prima in laboratorio. Nel caso non fosse possibile processare il medesimo entro 2 ore dal prelievo, è opportuno utilizzare tamponi con idonei terreni di trasporto (disponibili in commercio).

\section{Fase analitica}

La fase analitica vera e propria prevede a sua volta una prima fase di semina su idonei terreni di coltura agarizzati, ed una seconda di "lettura" di quanto può essere cresciuto sui medesimi. A questi seguirà necessariamente la meta-analisi dei risultati, ovvero l'interpretazione critica di quanto (e come) può essere cresciuto, l'esecuzione dell'antibiogramma, ossia la valutazione in vitro delle resistenze agli antibatterici, la refertazione finale, che potrà essere preceduta da eventuali comunicazioni preliminari (quando possibili e necessarie).

In Tabella IV riportiamo lo schema da seguire per la semina del materiale biologico pervenuto in laboratorio, raccolto mediante un tampone come già detto.

Si raccomandano due terreni non selettivi, entrambi idonei anche per eventuali germi esigenti e/o difficili: agar sangue e agar sangue cotto (agar cioccolato); il primo è preferibile incubarlo in normali condizioni di ossigenazione, il secondo in capnofilia (arricchimento di anidride carbonica).

La coltura è ottimale a $35^{\circ}-37^{\circ} \mathrm{C}$; l'incubazione va protratta per 72 ore, con l'avvertenza di osservazioni ogni 24 ore. Soltanto qualora l'esame batterioscopico risultasse significativo, può essere utile affiancare direttamente terreni agarizzạti selettivi e specifici al riguardo.

In Tabella $V$ riportiamo la chiave interpretativa della crescita delle colture microbiche (dopo 24, 48 o 72 ore). La cre-

TAB. III - RISULTATI DELLE INDAGINI PROSPETTICHE RELATIVE AI PRIMI 11 CASI PRESUNTI DI INFEZIONE DELL'EMERGENZA NEL CORSO DEL I SEMESTRE 1997

\begin{tabular}{|c|c|c|c|c|}
\hline \multicolumn{4}{|c|}{ INFEZIONI DELL'EMERGENZA } & \multirow{2}{*}{$\begin{array}{l}1997 \mathrm{PG} \\
\begin{array}{l}\text { Note } \\
\text { varie }\end{array}\end{array}$} \\
\hline $\begin{array}{l}\text { Caso } \\
\text { progressivo }\end{array}$ & $\begin{array}{l}\text { Coltura } \\
\text { diretta }\end{array}$ & $\begin{array}{l}\text { Coltura dopo } \\
\text { arricchimento }\end{array}$ & $\begin{array}{l}\text { Diagnosi } \\
\text { eziologica }\end{array}$ & \\
\hline 1 & +++ & POSITIVA & S. aureus & MET - S \\
\hline 2 & +++ & POSITIVA & C. striatum & $\mathrm{V}-\mathrm{S}$ \\
\hline 3 & NEGATIVA & POSITIVA & $\begin{array}{l}\text { E. faecalis } \\
\text { (contaminante?!) }\end{array}$ & $\mathrm{AM}-\mathrm{S}$ \\
\hline 4 & $\begin{array}{l}++-- \\
\text { (S. xylosus) }\end{array}$ & $\begin{array}{l}\text { POSITIVA } \\
\text { (S. xylosus) } \\
\text { (S. cohnii) }\end{array}$ & $\begin{array}{l}\text { S. xylosus } \\
\text { (S. cohnii MET-S) } \\
\text { (contaminante!?) }\end{array}$ & MET - S \\
\hline 5 & NEGATIVA & POSITIVA & $\begin{array}{l}\text { S. epidermidis } \\
\text { (contaminante?!) }\end{array}$ & MET - S \\
\hline 6 & ++- & POSITIVA & $\begin{array}{l}\text { S. xylosus } \\
\text { (dubbio?) }\end{array}$ & MET -S \\
\hline 7 & +++ & POSITIVA & S. aureus & MET - S \\
\hline 8 & +++ & POSITIVA & S. aureus & MET - S \\
\hline 9 & ++- & POSITIVA & $\begin{array}{l}\text { E. faecalis } \\
\text { Corynebacterium sp } \\
\text { (dubbio?) }\end{array}$ & $\begin{array}{l}A M-S \\
V-S\end{array}$ \\
\hline 10 & NEGATIVA & POSITIVA & $\begin{array}{l}\text { S. epidermidis } \\
\text { (contaminante) }\end{array}$ & MET-S \\
\hline 11 & +++ & POSITIVA & Corynebacterium sp & $V-S$ \\
\hline
\end{tabular}

scita di colonie solo sul I quadrante è da interpretarsi più spesso come contaminazione; analogamente la crescita sui primi due quadranti qualora la coltura risultasse decisamente mista. In entrambi i casi è prudente una ripetizione del prelievo (purtuttavia su supporto clinico). In tutti gli altri casi è d'obbligo procedere secondo gli abituali schemi microbiologici.

\section{Tunnel sottocutaneo}

L'infezione del tunnel può essere la conseguenza dell'infezione dell'emergenza lungo il tragitto sotto-cutaneo oppure può essersi formata localmente. I segni dell'infezione sono di solito più evidenti e la diagnosi potrebbe essere posta anche in assenza di secrezione vera e propria. Non avendo esperienza sufficiente al ri- guardo, vengono forniti suggerimenti che potrebbero essere rivisitati, nel caso nuovi spunti dovessero emergere nella pratica quotidiana così impostata.

\section{Fase pre-analitica}

Il tunnel, ovvero la porzione del catetere tra le due cuffie, va delicatamente sfilato prestando la massima attenzione alla sterilità. Il materiale inorganico va riposto in un recipiente sterile a bocca larga e inviato quanto prima, come tale, in laboratorio.

\section{Fase analitica}

In laboratorio verrà adottata, adattandola allo scopo, la tecnica di "Cleri”, come per i cateteri venosi centrali (18). In Tabella 
TAB. IV - ITER ANALITICO PER LA DIAGNOSI DI INFEZIONE DELL'EMERGENZA: FASE DELLA SEMINA

\section{DIAGNOSI EZIOLOGICA DI INFEZIONE DELL'EMERGENZA CUTANEA}

- Risospendere il tampone in un $\mathrm{ml}$ di soluzione fisiologica sterile

- Seminare con ansa calibrata per quadranti

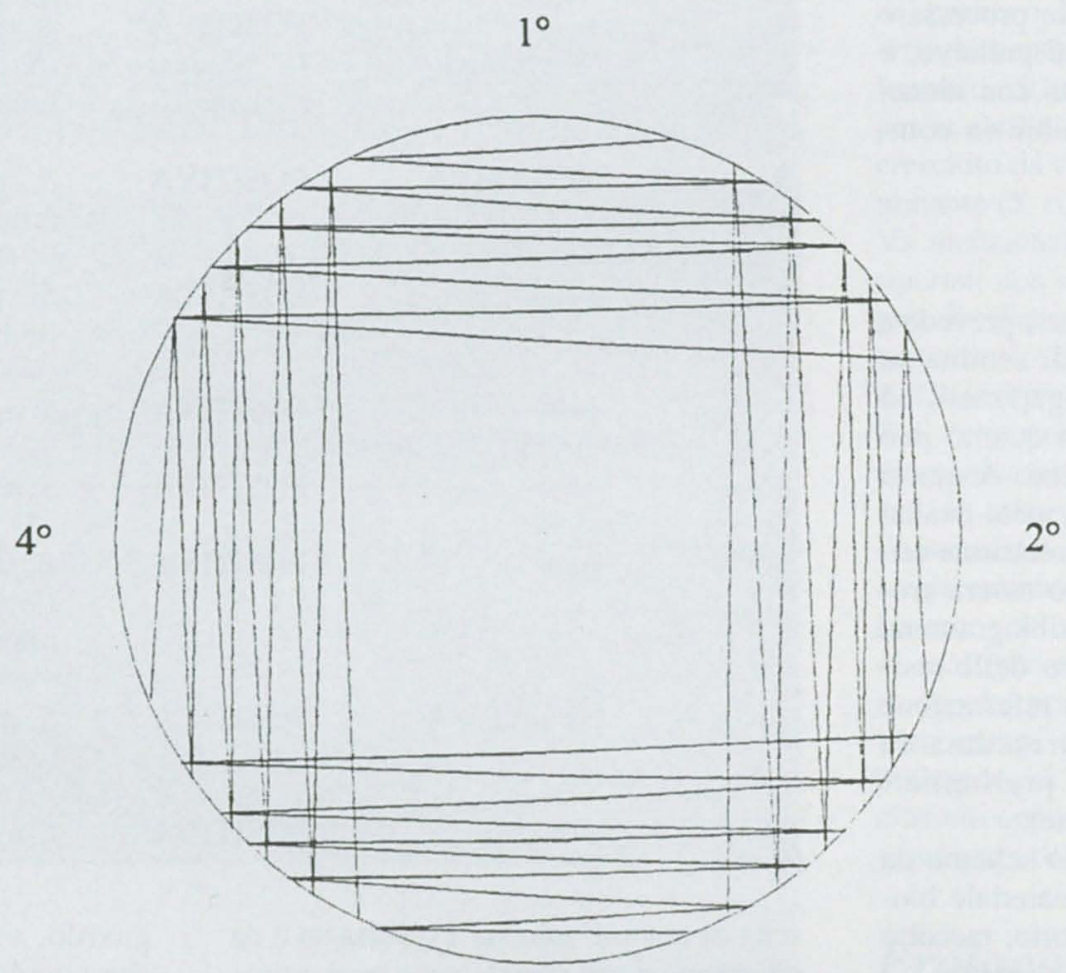

$3^{\circ}$

- utilizzare:

AGAR - SANGUE

$37^{\circ} \mathrm{C}+\mathrm{O}_{2}$

AGAR - CIOCCOLATO

$37^{\circ} \mathrm{C}+\mathrm{CO}_{2}$

- incubare:

$24-48-72$ ore

- in caso di materiale abbondante:

ESAME BATTERIOSCOPICO

(Colorazione secondo Gram) 
TAB. V - CHIAVE INTERPRETATIVA DELLA CRESCITA MICROBICA

\section{VALUTAZIONE SEMIQUANTITATIVA}

Crescita microbica

- nessuna

0

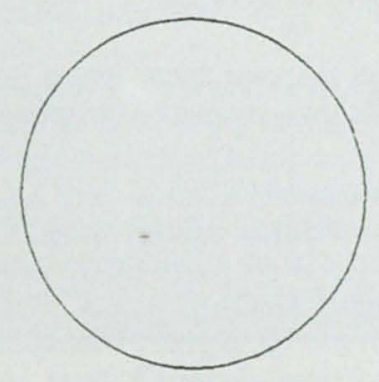

Esito colturale

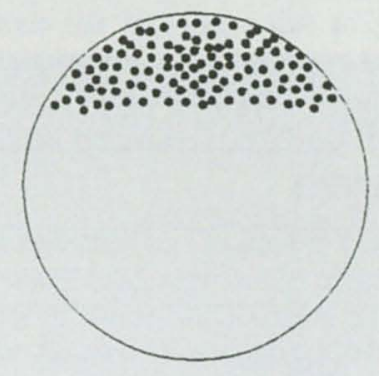

negativo

- sul I quadrante

$1+$

- sul II quadrante

$2+$

- sul III quadrante

$3+$

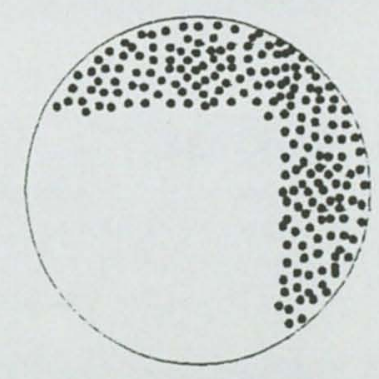

contaminazione

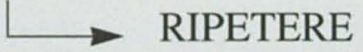

dubbio
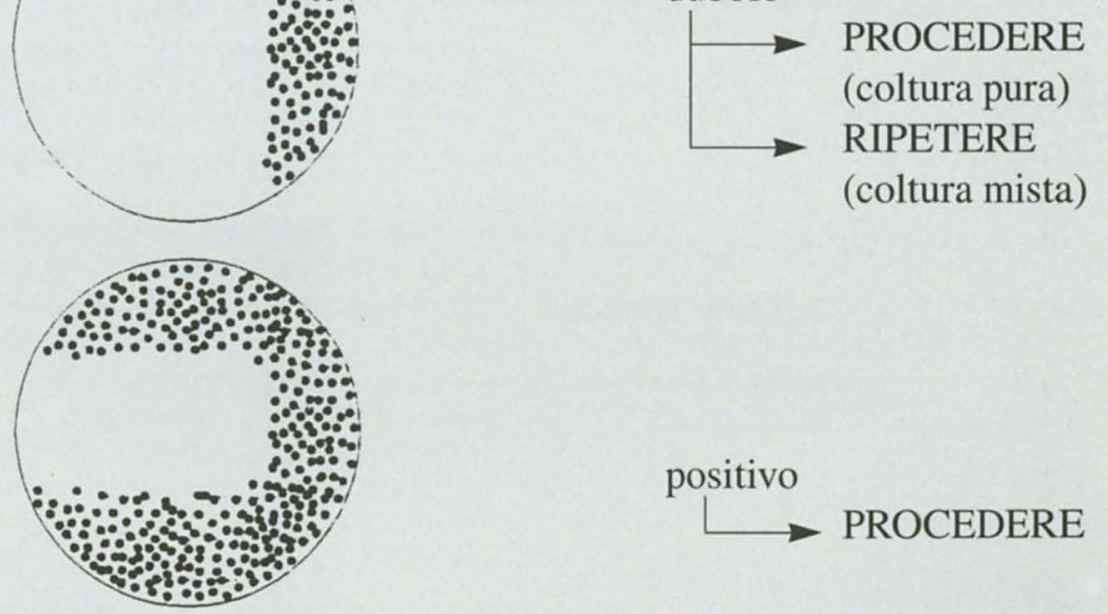

positivo

$\longrightarrow$ PROCEDERE

- sul IV quadrante

$4+$

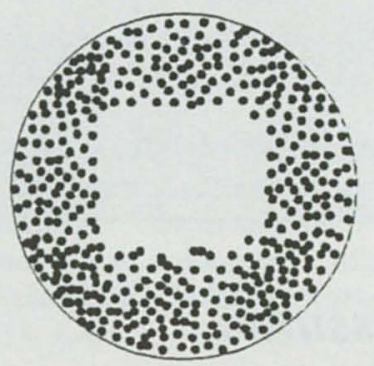

positivo

PROCEDERE 
TAB. VI - DIAGNOSI DI INFEZIONE DEL TUNNEL: FASE ANALITICA

\section{TUNNEL SOTTOCUTANEO}

\begin{tabular}{ll}
\hline - AGGIUNGERE & $5 \mathrm{ml}$ di brodo-triptosio \\
- AGITARE & su vortex ( 30 secondi) \\
- DEPOSITARE & $100 \mu \mathrm{l}$ di sospensione al centro \\
& della piastra agarizzata e spatolare \\
- utilizzare & - agar sangue $+\mathrm{O}_{2}$ \\
& - agar cioccolato $+\mathrm{CO}_{2}$ \\
- incubare & $24-72$ ore a $37^{\circ} \mathrm{C}$ \\
\hline
\end{tabular}

TAB. VII - INTERPRETAZIONI DELLA CRESCITA MICROBICA DALLA COLTURA DEL TUNNEL (TECNICA “CLERI” ADATTATA)

TUNNEL SOTTOCUTANEO

\section{Conteggio $n^{\circ}$ di colonie cresciute}

- inf. 10

$=\inf .10^{3}$

$\mathrm{UFC} / \mathrm{ml}$

verosimile contaminazione

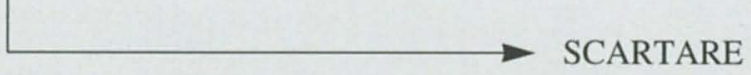

- $10-100$

$=10^{3}-10^{4}$

$\mathrm{UFC} / \mathrm{ml}$

probabile infezione

PROCEDERE

- sup. 100

$=$ sup. $-10^{4}$

$\mathrm{UFC} / \mathrm{ml}$

infezione certa

PROCEDERE te, seppure non risolte né del tutto risolvibili. Anche nella nostra esperienza, i cocchi Gram-positivi prevalgono quali agenti eziologici, in particolare Staphilococcus aureus, responsabile di circa la metà dei casi. Fortunatamente la meticillino-resistenza si attesta a valori inferiori al $40 \%$. Le difficoltà, peraltro, di correlare gli isolamenti in vitro alla reale situazione clinica pongono il microbiologo in prima linea per tracciare adeguati protocolli diagnostici, come in tale sede viene fatto, e per una continua sorveglianza del fenomeno.

Nella fattispecie compito del microbiologo clinico è quello di tracciare adeguate ed omogenee linee-guida atte a discriminare tra infezione e contaminazione, nonché supportare un monitoraggio attento e mirato in ambito di fenotipia e antibiotico-resistenza relativamente ai microorganismi isolati in sede di infezione.
VI si riportano le tappe della medesima. Terreni e modalità colturali sono analoghi a quelli descritti per l'emergenza cutanea. In tabella VII, infine, vengono riportate le chiavi interpretative.

\section{Riassunto}

Le problematiche relative alle infezioni dell'emergenza cutanea e del tunnel nei pazienti in CAPD sono ben documenta- 


\section{BIBLIOGRAFIA}

1.

Levy M, Balfe JW, Geary D, Fryer-Keene S, Bannatyne R. Exitsite Infection During Continuous and Cycling Peritoneal Dialysis in Children. Peritoneal Dialysis International 1990; 10: 31-5.

2. Scalamagna A, Castelnovo C, De Vecchi A, Ponticelli C. Exit-site and Tunnel Infections in Continuous Ambulatory Peritoneal Dialysis Patients. Am J Kidney Dis 1991; 18, 6: 674-7.

3. Luzar MA. Exit-site Infections in Continuous Ambulatory Peritoneal Dialysis: A Review. Peritoneal Dialysis International 1991; 11: $333-40$.

4. Georgi A, Savin E, Ayomamitis A, et al. Natural History of Exitsite Infection (ESI) in Patients on Continuous Ambulatory Peritoneal Dialysis (CAPD). Peritoneal Dialysis International 1988; 8: 211-6.

5. Luzar MA, Brown CB, Balf D, et al. Exit-site Care and Exit-site Infection in Continuous Ambulatory Peritoneal Dialysis (CAPD): Results of a Randomized Multicentral Trial. Peritoneal Dialysis International 1990; 10: 25-9.

6. Fattom AI, Naso R. Staphylococcus aureus. Vaccination for Dialysis Patients-An Update. Advances in Renal Replacement Therapy 1996; 3, 4: 302-8.

7. Davies SJ, Ogg CS, Cameron JS, Poston S, Noble WC. Staphylococcus aureus. Nasal Carriage, Exit-site Infection and Catheter Loss in $\mathrm{Pa}$ tients Treated with Continuous Ambulatory Peritoneal Dialysis (CAPD). Peritoneal Dialysis International 1989; 9: 61-4.

8. Turner $\mathrm{K}$, Edgar D, Hair M, et al. Does catheter immobilization reduce exit-site infection in CAPD patients? Adv Perit Dial 1992; 8: 265-8.

9. Han DC, Cha HK, So IN, et al. Subcutaneously implanted catheters re- duce the incidence of peritonitis during CAPD by eliminating infection by periluminal route. Adv Perit Dial 1992; 8: 298-301.

10. Carobi C, Gubbiotti G, Buoncristiani U, Crotti D. Microbiologia Medica Bollettino anno 16; 1: V-VII.

11. Piraino B, Bernardini J, Sorkin M. A Five-Year Study of the Microbiologic Results of Exit-site Infections and Peritonitis in Continuous Ambulatory Peritoneal Dialysis. Am J Kidney Dis 1987; 10, 4: 281-6.

12. Dapena F, Selgas R, Garcia-Perea $\mathrm{A}$, et al. Clinical significance of exit-site infections due to Xanthomonas in CAPD patients: a comparison with Pseudomonas infection. Nephrol Dial Transplant 1994; 9: 1774-7.

13. Lombardo V. Diagnosi e Trattamento delle infezioni dell'emergenza cutanea. Manuale di dialisi peritoneale. Gruppo Cooperativo per lo studio della CAPD. 1991; 35-44.

14. Zimmerman SW, O'Brien M, Wiedenhoeft FA, Johnson CA. Staphylococcus aureus. Peritoneal Catheter-Related Infections: A Cause of Loss and Peritonitis. Peritoneal Dialysis International 1988; 8: 191-4.

15. Khanna R, Twardoswki ZJ. Editorial. Peritoneal Catheter Exit-site. Peritoneal Dialysis International 1988; 8: 119-23.

16. Von Graevenitz A, Amsterdam D. Microbiological Aspects of Peritonitis Associated with Continuous Ambulatory Peritoneal Dialysis. Clinical Microbiology Reviews 1992; 5, 1: $36-48$

17. Twardowski ZJ. Exit-site infection. Peritoneal dialysis 1991; 241-5.

18. Rescaldani R, Giltri G, Bramati S, et al. Infezioni associate al cateterismo vascolare: Consensus sull'iter diagnostico microbiologico. Microbiologia Medica Bollettino anno 18; 1 : XIV-XXII. 